\section{Discussion of "Despiking Acoustic Doppler Velocimeter Data" by Derek G. Goring and Vladimir I. Nikora}

January 2002, Vol. 128, No. 1, pp. 117-126.

Tony L. Wahl ${ }^{1}$

${ }^{1}$ Hydraulic Engineer, U.S. Dept. of the Interior, Bureau of Reclamation, Water Resources Research Laboratory, Mail Code D-8560, P.O. Box 25007, Denver, CO 80225-0007. E-mail: twahl@ do.usbr.gov

The authors are commended for their novel approach to the difficult problem of removing spikes from velocity time series recorded by acoustic Doppler velocimeters (ADVs). Their paper has prompted some further investigations into modifications that may make the method even more practicable and robust.

To briefly review, spikes in ADV time series can be caused by many factors, including high turbulence intensities, aerated flows that have undesirable acoustic properties, and phase difference ambiguities that occur when velocities exceed the upper limits of ADV probe velocity ranges. Although spikes can be reduced or eliminated in many cases by adjustment of probe operational parameters, there are some situations in which spikes cannot be entirely avoided. A method for spike detection such as that proposed by the authors is thus necessary.

The phase-space thresholding method set forth by the authors is based on the observation that good ADV data are tightly clumped within an ellipsoid in phase space [three-dimensional (3D) plots of velocity, $u$, and approximations of the first and second derivatives, $\Delta u$ and $\Delta^{2} u$, respectively]. Spikes are those points plotting outside of elliptical projections of the ellipsoid onto the three principal phase-space planes, $u-\Delta u, \Delta u-\Delta^{2} u$, and $u-\Delta^{2} u$. The first two ellipses are symmetric about both axes, while the third is rotated due to correlation between $u$ and $\Delta^{2} u$; positive velocities are associated with negative second derivatives and vice versa as the velocity tends to move back toward its average value when an extreme is reached. The authors size the ellipsoid and the projected ellipses using the standard deviations of the $u, \Delta u$, and $\Delta^{2} u$ time series and a Universal Threshold parameter related to the length of the time series. The Universal Threshold requires no tuning, a fundamental advantage of the algorithm. The method is iterative, with spikes identified and replaced, and the algorithm repeated one or more times. Iteration is necessary because the standard deviations, and thus the ellipse boundaries, are sensitive to the spikes and change as spikes are identified and replaced. The authors point out that spike replacement is an arbitrary procedure with many strategies available. Spike replacement is necessary to use the iterative phase-space thresholding method, since derivative and second derivative estimates are needed in subsequent iterations.

\section{Identifying Points Outside of the Ellipsoid}

The use of two-dimensional (2D) ellipse projections requires that three comparisons be made to determine if a data point is a spike, one in each principal plane. For each comparison the distance of the data point from the center of the ellipse must be determined and compared to the corresponding distance to a point on the ellipse located along the ray connecting the center of the ellipse and the data point. Spikes are ultimately identified if they are located outside of a complex three-dimensional shape resembling an ellipsoid, but having knobby projections in each of the eight phase-space quadrants. This object is shown in Fig. 1 for the simplified case of three intersecting circular cylinders (i.e., standard deviations of $u, \Delta u$, and $\Delta^{2} u$ being identical), which produce a knobby sphere. The knobs allow some points that lie outside of the true ellipsoid to be retained as good data.

A single-comparison test using the true ellipsoid shape can identify spikes if the ellipsoid is defined in spherical coordinates, $\rho, \phi, \theta$ (Fig. 2)

$$
\begin{aligned}
\frac{1}{\rho^{2}}= & \frac{(\sin \phi \cos \theta \cos \alpha+\cos \phi \sin \alpha)^{2}}{a^{2}} \\
& +\frac{(\sin \phi \cos \theta \sin \alpha-\cos \phi \cos \alpha)^{2}}{b^{2}}+\frac{(\sin \phi \sin \theta)^{2}}{c^{2}}
\end{aligned}
$$

where $a$ and $b=$ major and minor axes of the ellipse projected onto the $u-\Delta^{2} u$ plane, as given by the authors' Eqs. (10) and (11); and $c=$ major axis in the $\Delta u-\Delta^{2} u$ projection, identified by the authors as $\lambda_{U} \sigma_{\Delta u}$. Here, the symbol $\alpha$ indicates the angle of rotation of the ellipsoid in the $u-\Delta^{2} u$ plane, given by the authors' Eq. (9). (The authors used the symbol $\theta$ for this parameter, which is being used here in connection with the spherical coordinate system.) The ellipsoid in Eq. (1) is centered at the origin, requiring one to remove the mean of the velocity time series before testing for spikes.

The test to identify points outside of the ellipsoid is performed in a manner similar to that described above. The position of the $u$, $\Delta u, \Delta^{2} u$ data point is expressed in spherical coordinates as a distance $\rho$ and the angles $\phi$ and $\theta$. The distance $\rho_{e}$ to a point on the surface of the ellipsoid at the same $\phi$ and $\theta$ coordinates is determined from Eq. (1) and $\rho$ and $\rho_{e}$ are compared to determine if the point is inside or outside the ellipsoid.

\section{Choice of Spike-Detection Threshold}

Although applied in 3D phase space, the method described by the authors is similar to one-dimensional methods for excluding out-
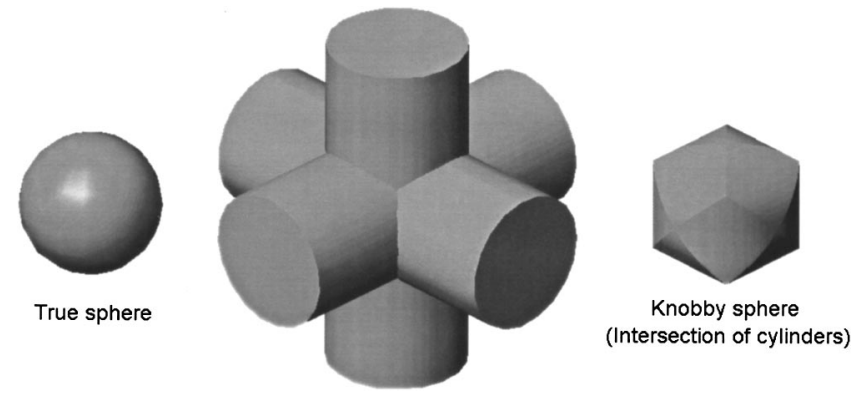

Fig. 1. True sphere and the object produced by the intersection of orthogonal circular cylinders 


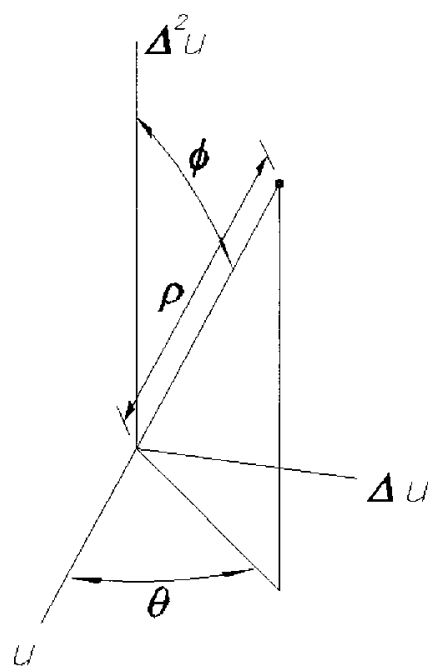

Fig. 2. Spherical coordinates and phase-space coordinates

liers from experimental data. Points lying outside of a threshold are marked as outliers and removed, or optionally replaced. The threshold used by the authors is $\sigma \sqrt{2 \ln (N)}$, in which $\sigma$ is the standard deviation and $N$ is the number of points. The easily calculated multiplier $\lambda_{U}=\sqrt{2 \ln (N)}$ is the Universal Threshold, described as the theoretical expected maximum value of $N$ independent samples drawn from a standard normal distribution. Thresholds are computed for the three phase-space axes, $u, \Delta u$, and $\Delta^{2} u$, and these values are used for the ellipse-based tests described earlier.

The authors noted that the Universal Threshold is about $10 \%$ larger than expected for $N>1,000$. If the threshold were truly the expected maximum value of $N$ independent samples, then we should expect that the performance of the exclusion method would be independent of the value of $N$. To test this, the expected number of observations rejected can be determined by computing the threshold, $\sqrt{2 \ln (N)}$, and determining the corresponding rejection probability from a two-tailed standard-normal probability table. The expected number of rejections varies slightly with $N$, as shown in Fig. 3, starting at about 0.5 and decreasing as $N$ increases.

Another common method for excluding outliers from experimental data uses Chauvenet's criterion (Coleman and Steele

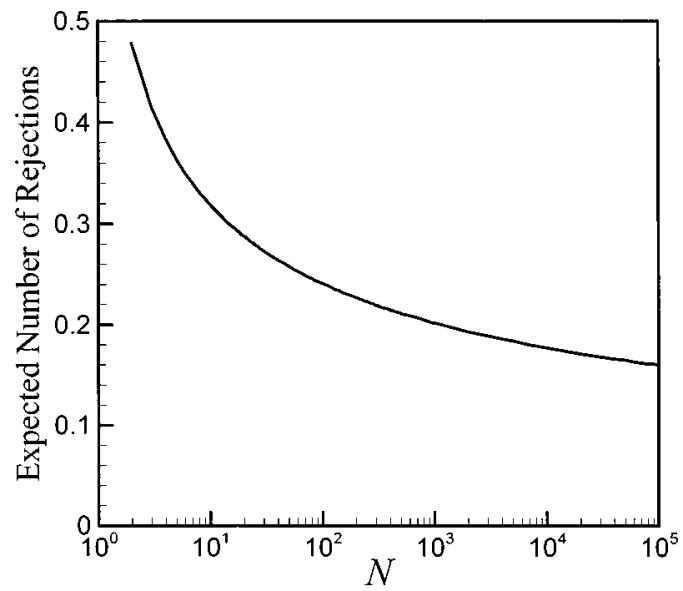

Fig. 3. Expected number of rejections from a normally distributed data set of $N$ elements, using the Universal Threshold
1999). For a set of $N$ data, a rejection probability is computed, $p=1 /(2 N)$, and the $Z$ statistic for this probability is determined from a two-tailed normal probability table. The $Z$ statistic for each observation is then computed, $Z=(x-\mu) / \sigma$. Those observations outside of the $\pm Z$ range are excluded. The expected number of observations excluded is always $N /(2 N)$, or 0.5 , assuming the data are drawn from a standard-normal distribution. This approach has the property we expected for the Universal Threshold; the performance is independent of the size of the sample, and we always stand a coin-flip chance of excluding one observation from a true normally distributed data set. Chauvenet's criterion should be applied only once to a given data set.

For an automated despiking algorithm, an easily calculated threshold is desirable. Chauvenet's criterion can be determined without using lookup tables, since standard-normal probability values can be computed using the error function, $\operatorname{erf}(x)$, determined by series approximation (Kreyszig 1988)

$\operatorname{erf}(x)=\frac{2}{\sqrt{\pi}}\left[x-\frac{x^{3}}{1 ! 3}+\frac{x^{5}}{2 ! 5}-\frac{x^{7}}{3 ! 7}+\left.\left((-1)^{j} \frac{x^{2 j+1}}{j !(2 j+1)}\right)\right|_{j=4} ^{j=\infty} \ldots\right]$

Eq. (2) converges quickly for small values of $x$, but may require $j=30-40$ terms for $x>5$. The rejection probability for the twotailed case for a standardized observation, $Z=(x-\mu) / \sigma$ is $p$ $=1-\operatorname{erf}(Z / \sqrt{2})$ and the $Z$ statistic for a given rejection probability is $Z=\sqrt{2} \operatorname{erfinv}(1-p)$. The inverse of the error function, $\operatorname{erfinv}(1-p)$, can be determined by Newton's method making use of subroutines that return the error function and its derivative.

\section{Robustness of the Despiking Threshold}

Regardless of the threshold chosen, a method that is insensitive to the outliers themselves is desirable. The despiking algorithm presented by the authors uses the mean and standard deviation, the classic estimators for location (general position of the data) and scale (spread of the data), respectively. Rousseeuw (1998) shows that both are very sensitive to outliers, having a breakdown point of $1 / N$. The breakdown point is the smallest fraction of the values that must be replaced with outliers to carry the estimator over all bounds. One may choose which observations to replace, as well as the magnitude of the outliers, in the most unfavorable way.

When outlier exclusion is based on the mean and standard deviation, a single outlier of extraordinary magnitude can corrupt both parameters and significantly affect performance. The location estimator moves toward the outlier and away from the good data, and the scale estimator explodes, protecting the outliers from rejection. If outlier exclusion is performed iteratively, the result will improve as outliers are excluded, assuming that the initial iterations exclude some outliers and are not so seriously affected that they exclude nonoutlying data.

Outlier exclusion can be improved by using robust location and scale estimators. A robust estimator of location is the sample median. The median has the largest possible breakdown point, $50 \%$, meaning that one must replace at least half the values to ensure that the estimator will exceed the range of the original data. An extremely robust estimator of scale is based upon the median of the absolute deviations (MAD) from the sample median,

$$
S=1.483\left(\operatorname{median}_{j=1, \ldots, n}\left|x_{j}-\operatorname{median}_{i=1, \ldots, n}\left(x_{i}\right)\right|\right)=1.483(\text { MAD) }
$$

in which $1.483=$ factor that makes the estimator analogous to the standard deviation, the usual scale parameter of a normal distribution. This estimator also has a breakdown point of $50 \%$. 
Table 1. Comparison of Despiking Methods

\begin{tabular}{|c|c|c|}
\hline & Contaminated record & Clean record \\
\hline Length of time series & 30,000 samples & 30,000 samples \\
\hline \multicolumn{3}{|l|}{ Goring-Nikora method (Universal Threshold) } \\
\hline Standard deviation of $u$ before despiking & $23.52 \mathrm{~cm} / \mathrm{s}$ & $8.058 \mathrm{~cm} / \mathrm{s}$ \\
\hline Spikes identified in $u$ in first iteration & 858 & 14 \\
\hline $\begin{array}{l}\text { Standard deviation of } u \text { after one iteration (spikes removed, } \\
\text { but not replaced) }\end{array}$ & $14.68 \mathrm{~cm} / \mathrm{s}$ & $8.050 \mathrm{~cm} / \mathrm{s}$ \\
\hline $\begin{array}{l}\text { Standard deviation of } u \text { after four iterations (spikes replaced } \\
\text { using cubic fit) }\end{array}$ & $13.78 \mathrm{~cm} / \mathrm{s}$ & $\cdots$ \\
\hline \multicolumn{3}{|c|}{ Effect of 3-D ellipsoid test [Eq. (1)] and identifying spikes in all velocity components } \\
\hline Spikes in $u$ in first iteration & 880 & 20 \\
\hline Additional spikes in $v$ and $w$ time series & 229 & 160 \\
\hline \multicolumn{3}{|c|}{ Robust despiking algorithm using MAD, Chauvenet's criterion, 3D ellipsoid test, and all velocity components } \\
\hline 1.483(MAD) of $u$ before despiking & $13.55 \mathrm{~cm} / \mathrm{s}$ & $7.904 \mathrm{~cm} / \mathrm{s}$ \\
\hline Spikes identified in $u$ time series & 2,149 & 49 \\
\hline Additional spikes in $v$ and $w$ time series & 474 & 415 \\
\hline 1.483(MAD) of $u$ after despiking & $12.89 \mathrm{~cm} / \mathrm{s}$ & $7.845 \mathrm{~cm} / \mathrm{s}$ \\
\hline Standard deviation of $u$ after despiking & $13.19 \mathrm{~cm} / \mathrm{s}$ & $7.994 \mathrm{~cm} / \mathrm{s}$ \\
\hline
\end{tabular}

An outlier-exclusion algorithm described by Rousseeuw (1998) computes the standardized observations, $Z_{i}=\left(x_{i}-T\right) / S$. The median is used for the location estimator, $T$, and the scale estimator, $S$, is based upon the MAD as shown in Eq. (3). The absolute values of the standardized observations are compared to a cutoff value, somewhat arbitrarily chosen as 2.5 , and observations above the cutoff are outliers. The cutoff could also be established using the Universal Threshold or Chauvenet's criterion. Regardless, the algorithm need only be applied once, as the estimators of location and scale will change only slightly after removal of the outliers.

\section{Application to Multidimensional Velocity Time Series}

The phase-space threshold despiking method is described by the authors for application to the time series of a single component of velocity. Most ADV probes report three-dimensional velocities (or 2D in some cases). The reported orthogonal velocity components are interdependent, since the instrument actually measures beam velocities along the bistatic axes of the sending and receiving acoustic elements and converts these to orthogonal velocity components via multiplication by a transformation matrix (Kraus et al. 1994). Factors that produce spikes often affect only one of the individual beam velocities (e.g., overranging caused by a large turbulent velocity burst aligned with one of the bistatic axes, or a single air bubble corrupting one acoustic beam), but after multiplication by the transformation matrix, the single affected beam velocity taints all three of the orthogonal velocity components. As a result, a conservative filtering algorithm should eliminate and/or replace associated data points in all three time series when a spike is identified in any one time series, even if the data in the other time series do not appear to be spikes.

\section{Robust Phase-Space Despiking Algorithm}

A despiking algorithm based on the authors' phase-space thresholding method and incorporating the concepts discussed above was developed and added to the WinADV computer program, a freely distributed software package used for postprocessing and analysis of ADV data (Wahl 2000). WinADV was developed primarily for use by the Bureau of Reclamation's Water Resources Research Laboratory, where ADV's are used to collect laboratory and field data around model and prototype hydraulic structures and in reservoirs and natural channels. The primary objective of these measurements is usually to define average velocities and turbulence parameters in two or three dimensions.

The modified algorithm incorporates the following features to create a robust, conservative despiking method whose performance is independent of the length of the ADV time series.

- The median and MAD are used as location and scale estimators.

- Chauvenet's criterion is used to define the rejection probability and exclusion thresholds (principal axes of the ellipsoid in phase space).

- The exclusion test uses the true ellipsoid shape defined in spherical coordinates.

- Despiking is performed on each of the available velocity components (usually three), and all associated data are removed when a spike is detected in any one of the time series.

Before despiking, the median of each velocity time series is removed. Alternatively, one could high-pass filter the data as described by the authors. Spike replacement is not used, since the algorithm is no longer iterative and does not require replacement of spikes. For most applications of ADV's at the Bureau of Reclamation, a continuous time series is not needed, and a data set that does not contain synthesized or interpolated data is desirable. 


\section{Example Application}

The method described above was applied to the two data sets examined by the authors. The results are compared in Table 1 to the authors' method. The modified method identifies more spikes than the authors' method in three different ways:

- excluding data within the knobs of the "knobby" ellipsoid;

- reducing the size of the ellipsoid by using the MAD as the scale estimator and Chauvenet's criterion to define the rejection probability and exclusion thresholds; and

- examining all velocity components and rejecting associated data in all time series when a spike is detected in any one component.

\section{Analysis}

The modifications to the authors' method each identify more spikes, with the greatest effects in these examples being caused by the reduction of the exclusion thresholds and the examination of all three associated time series. The number of additional spikes located in the knobs of the knobby ellipsoid was relatively small.

In the contaminated data set, examination of the $v$ and $w$ velocity components added about $25 \%$ more spikes than were detected in the $u$ component, and of these, about two thirds came from the $w$ component. In the clean data set, the $v$ and $w$ components added about eight times more spikes than were found in the $u$ component alone, and about $85 \%$ were due to the $w$ component. These results were similar, regardless of which threshold was used (the Universal Threshold or the MAD and Chauvenet's criterion). The relatively large number of additional spikes found in the $w$ component may be an anomaly of these two data sets; the authors reported that in their experience with down-looking ADV probes the vertical component ( $w$ in these cases) usually contains far fewer spikes than the horizontal components.

Using the MAD and Chauvenet's criterion to define the rejection thresholds significantly increases the number of spikes in comparison to the first iteration of the authors' method, although subsequent iterations would identify additional spikes. The advantages of the approach utilizing the MAD are the robustness and insensitivity to the spikes themselves, and the fact that despiking need not be done iteratively.

The methods can also be evaluated by comparing the standard deviations and median absolute deviations before and after despiking, shown in Table 1. In the clean record, the standard deviations and the values of 1.483(MAD) are similar before and after despiking. This is expected, since the outliers in this data set are few in number and not extreme. In the contaminated record (which the authors aptly describe as pathological), the standard deviation of the $u$ time series is reduced dramatically from 23.52 to $14.68 \mathrm{~cm} / \mathrm{s}$ by the first iteration of the authors' method. Four iterations of despiking and spike replacement reduce the standard deviation to a final value of $13.78 \mathrm{~cm} / \mathrm{s}$. In comparison, the value of 1.483 (MAD) is $13.55 \mathrm{~cm} / \mathrm{s}$ before despiking, comparable to the standard deviation after despiking. The robust despiking method reduces the value modestly to $1.483(\mathrm{MAD})=12.89 \mathrm{~cm} / \mathrm{s}$, demonstrating that this scale estimator is relatively insensitive to spikes and is a reasonable approximation to the standard deviation. The final standard deviation of $u$ after applying the robust despiking method is $13.19 \mathrm{~cm} / \mathrm{s}$.

\section{References}

Coleman, H. W., and Steele, W. G. (1999). Experimentation and uncertainty analysis for engineers, 2nd Ed., Wiley, New York, 34-37.
Kraus, N. C., Lohrmann, A., and Cabrera, R. (1994). "New acoustic meter for measuring 3D laboratory flows." J. Hydraul. Eng., 120(3), 406-412.

Kreyszig, E. (1988). Advanced engineering mathematics, 6th Ed., Wiley, New York, A78.

Rousseeuw, P. J. (1998). "Robust estimation and identifying outliers." Handbook of statistical methods for engineers and scientists, 2nd Ed., H. M. Wadsworth Jr., ed., McGraw-Hill, New York, 17.1-17.15.

Wahl, T. L. (2000). "Analyzing ADV data using WinADV." Proc., 2000 Joint Conference on Water Resources Engineering and Water Resources Planning and Management, American Society of Civil Engineers, July 30-August 2, Minneapolis, www.usbr.gov/wrrl/twahl/winadv

\section{Closure to "Depiking Acoustic Doppler Velocimeter Data" by Derek G. Goring and Vladimir I. Nikora}

January 2002, Vol. 128, No. 1, pp. 117-126.

\section{Derek G. Goring ${ }^{1}$ and Vladimir I. Nikora ${ }^{2}$}

${ }^{1}$ Principal Scientist, National Institute of Water and Atmospheric Research, P.O. Box 8602, Christchurch, New Zealand. E-mail: d.goring@niwa.co.nz

${ }^{2}$ Principal Science, National Institute of Water and Atmospheric Research, P.O. Box 8602, Christchurch, New Zealand.

We thank the discussor for considering our phase-space thresholding method for application in his excellent WinADV program (that we routinely use for our first look at ADV data) and commend him for making suggestions for improvements to the algorithm. In this Closure, we consider each of the points he raises in the Discussion (most of which we agree with) and place our interpretation on them.

\section{Three-Dimensional Ellipsoid}

The discussor suggests that instead of using three projections of the ellipsoid in two-dimensional (2D) space, we should identify the spikes in three-dimensional (3D) space. We believe these approaches are exactly the same, and this can be illustrated by considering how one calculates the angles $\phi$ and $\theta$ in Fig. 2 of the discussion. To calculate $\theta$, we project the point onto the $u-\Delta u$ plane and calculate

$$
\theta=\tan ^{-1} \frac{\Delta u}{u}
$$

then for $\phi$ we rotate the vertical $u-\Delta^{2} u$ plane by $\theta$ and calculate

$$
\phi=\tan ^{-1} \frac{\sqrt{u^{2}+\Delta u^{2}}}{\Delta^{2} u}
$$

Whether we project the ellipsoid onto 2D space and look for points outside (writers' algorithm), or project the points onto $2 \mathrm{D}$ space, define their latitude and longitude, and test whether they lie inside the ellipsoid in 3D space (discussor's algorithm), makes no difference. The answers are exactly the same.

\section{Threshold}

The discussor shows that using the normal distribution, the expected number of rejections using the Universal Threshold varies 
inversely with the number of data $N$, with the rejection being about 0.5 for $N=2$. But for all practical purposes with acoustic Doppler velocimeters (ADV) data, $N$ will be in the range from $10^{4}$ to $10^{7}$, corresponding to rejections from 0.177 to 0.137 , which for all intents and purposes are the same. The discussor states that an easily calculated threshold is desirable, and we agree, but assert that the Universal Threshold $\lambda_{U}=\sqrt{2 \ln n}$ is very easily calculated and for practical numbers of data gives consistent results.

\section{Robustness}

The discussor proposes the use of median of the absolute deviations (MAD) for the scale parameter of the threshold. We are grateful to the discussor for this suggestion. It is indeed a more robust parameter than the standard deviation. However, use of MAD does not obviate the need for iteration. Indeed, iteration is undertaken not only because of changing thresholds, but also for spike replacement, especially for multipoint spikes as indicated in Fig. 5, and to realign the ellipse in the $u-\Delta^{2} u$ projection.

\section{Spike Replacement}

The discussor does not use spike replacement and we assume he simply eliminates the spikes from the sequence, thereby reducing the length of the record by the number of spikes. This may be a satisfactory strategy when computing bulk statistical moments, but we would like to suggest that for any other calculations (such as spectra, correlations, structure functions, or fractal analysis) this strategy is unsatisfactory because it changes the structure of the signal. Almost any spike replacement method is preferable to spike elimination.

\section{High-Pass Filtering}

The purpose of high-pass filtering was not understood by the discussor, and we have had several other enquiries about it. We welcome the opportunity to clarify this point.

High-pass filtering is required if the time series has longperiod oscillations that may dominate the turbulence. To illustrate this point, consider the portion of clean record from Fig. 3(a) that is shown in Fig. 1 of this closure. The signal has been separated into its high- and low-pass parts, by performing wavelet decomposition using the Daubechies No. 5 mother wavelet to level 6 (corresponding to timescales of about $1 \mathrm{~s}$ and less), then reconstituting and combining the 6 wavelet details. The low-pass filtered portion is the wavelet approximation (i.e., the remainder) at level 6. For a signal such as Fig. 1(a), the scale factor used in the threshold [Eq. (2)], whether it be the standard deviation or the MAD, is dominated by the low-frequency part of the signal and spikes (such as the one at $151 \mathrm{~s}$ ) can go undetected because they are hidden within the low-frequency oscillations. High-pass filtering exposes the spikes, so that they can be dealt to.

\section{Second Derivative}

The second derivative defined by Eq. (3) is based on central differences of the first derivative, which is also calculated by central differences. Thus, the full expression for the second derivative increment is

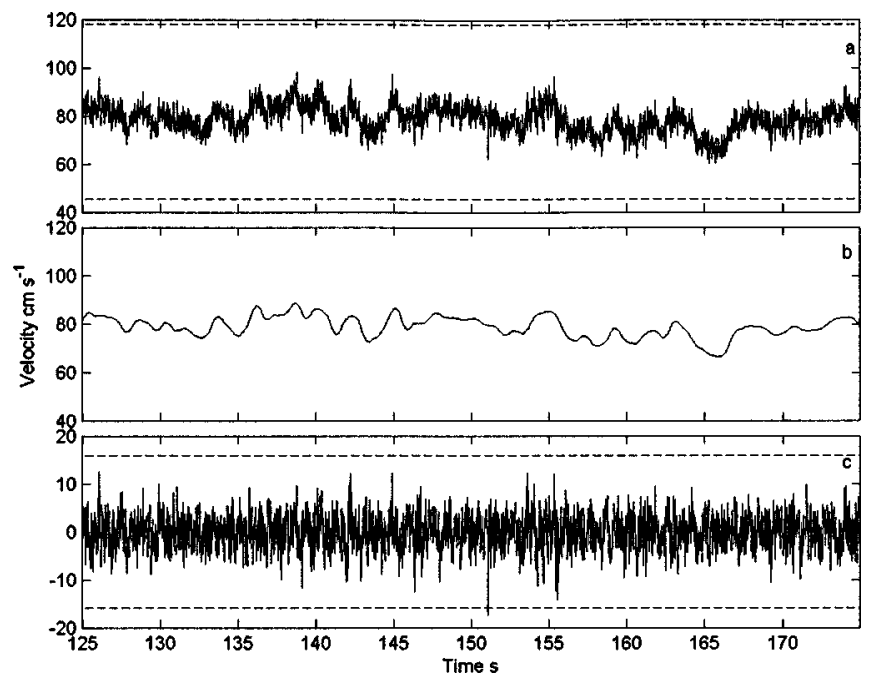

Fig. 1. Portion of the velocity series in Fig. 3: (a) raw data; (b) low-pass filtered part; and (c) high-pass filtered part. Dashed lines are the Universal Threshold.

$$
\Delta^{2} u_{i}=\left(u_{i+2}-2 u_{i}+u_{i-2}\right) / 4
$$

which excludes the points adjacent to the point under consideration. An alternative, and equally valid, definition for the second derivative increment is

$$
\Delta^{2} u_{i}=u_{i+1}-2 u_{i}+u_{i-1}
$$

which is the quadratic definition. Including the points adjacent to a spike intuitively would seem to improve the algorithm. However, for the contaminated velocity sequence shown in Fig. 3(a), fewer spike events were detected using Eq. (2) than Eq. (1) (622 versus 858). The main effect of applying Eq. (2) appears to be to change the relationship between the second derivative and the other components. Scaling Eq. (2) by 0.5 increased the number of spike events detected to 735 (0.5 appeared to be the optimum scaling to maximize the number of spike events), but this is still short of the number detected using Eq. (1). Another effect of using Eq. (2) instead of Eq. (1) is that the solution of Eqs. (9)(11) becomes more problematical, because $\theta$, the rotation angle of the ellipse in the $u-\Delta^{2} u$ projection, can take a larger range of values, necessitating the major and minor axes to be swapped if the following relationships are not satisfied:

$$
\begin{aligned}
& \text { For } \tan ^{2} \theta>1, \beta^{2} \tan ^{2} \theta>1 \quad \text { and } \beta^{2}<\tan ^{2} \theta \\
& \text { For } \tan ^{2} \theta<1, \beta^{2} \tan ^{2} \theta<1 \quad \text { and } \beta^{2}>\tan ^{2} \theta
\end{aligned}
$$

where $\beta=$ ratio of the thresholds, $\sigma_{\Delta^{2}{ }_{u}} / \sigma_{u}$.

\section{Data Availability}

Finally, we welcome others to test the performance of their despiking algorithm against our 3D phase space method, and to this end, we are pleased to make the clean and contaminated datasets used in this work available to all researchers. The data can be obtained by anonymous ftp on ftp.niwa.co.nz, cd incoming/ despiking. 


\section{Discussion of "Bibliography for Teaching Hydraulic Design" by ASCE Task Committee on Teaching of Hydraulic Design}

December 2001, Vol. 127, No. 12, pp. 1052-1064.

\section{Jack Cook, M.ASCE, ${ }^{1}$ and Thomas Walski, M.ASCE ${ }^{2}$}

${ }^{1}$ Chief Scientist, Haestad Methods, Inc., 37 Brookside Rd., Waterbury, CT 06708. E-mail: jcoo@haestad.com

${ }^{2}$ Vice President Engineering, Haestad Methods, Inc., 37 Brookside Rd., Waterbury, CT 06708. E-mail: twal@haestad.com

Developing a bibliography of hydraulic design is a formidable task and we congratulate the committee for attempting it; however, the authors made a significant oversight when they omitted Haestad Methods, Inc. from the list of commercial organizations whose works were cited.

Haestad Methods, Inc.'s, Computer Applications in Hydraulic Engineering (1997) is the most widely used hydraulics and hydrology textbook in the world today. It has sold over 18,000 copies and has been adopted by 90 schools worldwide and is now in its fifth edition. The book Water Distribution Modeling (Walski et al. 2001) is widely acclaimed as one of the most useful textbooks in the field and sold over 3,000 copies in its first months of publication. In addition, Haestad Methods, Inc., has reached roughly 1,500 hydraulics students in 2001, making it larger than any university. The classes covered hydraulic design subjects ranging from water distribution and pond routing to sewer design and flood plain mapping.

These references are widely used in both education and engineering practice and should have been mentioned in Sec. 3.8.

\section{References}

Haestad Methods, Inc. (1997). Computer applications in hydraulic engineering, Haestad Methods, Inc., Waterbury, Conn.

Walski, T. M., Chase, D. V., and Savic, D. (2001). Water distribution modeling, Haestad Methods, Inc., Waterbury, Conn.

\section{Closure to "Bibliography for Teaching Hydraulic Design" by ASCE Task Committee on Teaching of Hydraulic Design}

December 2001, Vol. 127, No. 12, pp. 1052-1064.

John Finnie ${ }^{1}$; Kevin Nielsen²; Walt Heyder ${ }^{3}$; and

Rollin Hotchkiss ${ }^{4}$

${ }^{1}$ Chair and Professor, Dept. of Civil Engineering, West Virginia Univ. Institute of Technology, 405 Fayette Pike, Montgomery, WV 25136. E-mail: jfinnie@wvutech.edu

${ }^{2}$ Assistant Professor, Dept. of Civil Engineering, Carroll College, 1601 North Benton Ave., Helena, MT 59625-002. E-mail: knielsen@carroll.edu

${ }^{3}$ Civil Engineer, Waterway and Concrete Dams Group, Bureau of Reclamation, Denver Federal Center, P.O. Box 25007 (D-8130), Denver, CO 80225. E-mail: wheyder@do.usbr.gov

${ }^{4}$ Associate Professor, Albrook Hydrology Laboratory, Civil and Environmental Engineering Dept., Washington State Univ., P.O. Box 642910 , Pullman, WA 99164-2910. E-mail: rhh@wsu.edu

Thank you for your discussion of our article.

In selecting material for the bibliography, we focused on works which presented design information. As a result, some books were omitted from the bibliography. These included text and other books which, for the most part, presented information about hydraulics theory and analysis. Your book is in a special category, in that it presents hydraulic theory and shows how to apply your commercial software to analyze and design hydraulic problems.

We recognize the important role played by software in today's engineering environment. The widespread use of your book is an indication of that importance. Thanks for bringing it to our attention. 